\title{
THE REFINED THEORY OF ELASTIC PLATES AS ASYMPTOTIC APPROACH OF 3D PROBLEM
}

\author{
Nelly Rogacheva ${ }^{1, *}$ \\ ${ }^{1}$ Moscow State University of Civil Engineering, Yaroslavskoe shosse, 26, Moscow, 129337, Russia
}

\begin{abstract}
The refined theory of elastic thin and thick plates is constructed by the asymptotic method for reducing three-dimensional (3D) equations of linear elasticity to two-dimensional ones without the use of any assumptions. The resulting refined theory is much more complicated than the known classical Kirchhoff theory: the required values of the refined theory vary in thickness of the plate by more complex laws; the system of partial differential equations of the refined theory has a higher order than the system of equations of the classical theory. A comparison of the obtained theory with the popular refined theory of Timoshenko and E. Reissner, taking into account the transverse shear deformation is made. It is shown that the inclusion only of the transverse shear deformation is insufficient. In addition to the transverse shear deformation, many additional terms having the same order as the transverse shear deformation must be taken into account.
\end{abstract}

\section{Introduction}

Elastic plates are widely used in various areas of modern engineering. For example, in civil construction plates are used as floors, walls, slab foundations, etc. The solution of threedimensional problems of elasticity for domains in which one dimension is much less than the others is associated with mathematical difficulties; therefore, such $3 \mathrm{D}$ problems are commonly replaced by approximate 2D problems of elasticity containing two variables instead of three independent variables. We note two methods for constructing twodimensional theories. The first method is the method of hypotheses. Well known Kirchhoff's plate theory is constructed by the use of this method. Kirchhoff used several physically understandable assumptions, analogous to the assumptions applied in the theory of beams. Hypotheses make an unavoidable error in the equations of the theory of plates. The first rough estimate of the error in the classical theory of plates was obtained in [1], where it was shown that the error of Kirchhoff's plate theory is the value of the order of $h / R$ ( $R$ is the characteristic size of the plate, $h$ is its half - thickness). Later, by a mathematical method, the following valid estimate of the error in Kirchhoff's theory of plates was obtained [2]. It is equal to

$$
\varepsilon_{k}=(h / R)^{2-2 s}
$$

\footnotetext{
* Corresponding author: nrogache@yandex.ru
} 
where $s$ is the variability of the stress-strain state or characteristic length of the strain pattern.

Another method of constructing the theory of plates is the asymptotic method, which makes it possible to use the smallest thickness of the plate most fully. . The implementation of the asymptotic method consists of the following steps: we perform a scale extension with respect to independent variables in $3 \mathrm{D}$ equations of the theory of elasticity in such a way that differentiation with respect to them does not lead to a significant increase or decrease in the unknown functions; then we write all the unknown quantities in a dimensionless form and find the asymptotic orders of the unknown quantities relative to each other. The asymptotic order of each quantity is written in the form of a relative half- thickness of the plate to some power. The asymptotic representation of the required quantities reflects the analytical nature of the problem, allows to deal with the values of the same order at each stage of the problem, to build the theory of plates with a given accuracy. It is known that the problem of linear elasticity theory has a unique solution. This unique solution corresponds to a unique asymptotic representation. If the asymptotic representation is chosen incorrectly, it will lead to an erroneous plate theory with obvious contradictions. Performing in the 3D equations of elasticity theory, asymptotic stretching of the scale along the coordinate lines and taking into account the asymptotic representation of desired quantities we reduce $3 \mathrm{D}$ equations to equations in the theory of plates.

\section{The basic equations}

The plate is placed in Cartesian coordinate system. The coordinate axes $x_{1}$ and $x_{2}$ lie in the middle plane of the plate and axis $x_{3}$ is normal to them.

A complete system of equations in the theory of elasticity consists of the equilibrium equations, the geometrical relations between the components of strain tensor $e_{n m}$ and the components displacement vector $u_{n}$, constitutive relationships (Hooke's law) for stresses $\sigma_{n m}$ and strains $e_{n m}$.

The system of equations may be written as

The equilibrium equations

$$
\frac{\partial \sigma_{i i}}{\partial x_{i}}+\frac{\partial \sigma_{i j}}{\partial x_{j}}+\frac{\partial \sigma_{i 3}}{\partial x_{3}}=0, \quad \frac{\partial \sigma_{13}}{\partial x_{1}}+\frac{\partial \sigma_{23}}{\partial x_{2}}+\frac{\partial \sigma_{3}}{\partial x_{3}}=0 ;
$$

The strain-displacement formulas

$$
e_{i i}=\frac{\partial u_{i}}{\partial x_{i}}, e_{i j}=\frac{\partial u_{j}}{\partial x_{i}}+\frac{\partial u_{i}}{\partial x_{j}}, e_{i 3}=\frac{\partial u_{i}}{\partial x_{3}}+\frac{\partial u_{3}}{\partial x_{i}}, e_{33}=\frac{\partial u_{3}}{\partial x_{3}}
$$

Hooke's law is written in an easy-to-use form solving according to $\sigma_{11}$ and $\sigma_{22}$

$$
\sigma_{i i}=\frac{E}{1-v^{2}}\left(e_{i i}+v e_{j j}\right)+\frac{v}{1-v} \sigma_{33}, \sigma_{i j}=\frac{E}{2(1+v)} e_{i j}, E e_{33}=\sigma_{33}-v\left(\sigma_{11}+\sigma_{22}\right), e_{i 3}=\frac{2(1+v)}{E} \sigma_{i 3} .
$$

Mechanical boundary conditions for stresses on the external faces take the following form:

$$
\left.\sigma_{n 3}^{(N)}\right|_{x_{3}= \pm h}= \pm q_{n}^{ \pm}
$$

Here $(i \neq j)=1,2$ and $\mathrm{n}=1,2,3$. 
It is assumed that the surface load vector with components $q_{1}^{+}, q_{2}^{+}, q_{3}^{+}$is given at any points of the external plate's face $x_{3}=h$, and the surface load vector with components $q_{1}^{-}, q_{2}^{-}, q_{3}^{-}$is given at any points of the external plate's face $x_{3}=-h$. Hereafter any formula with the \pm sign contains two equations; one is obtained by deletion all minus signs, and the other is by deletion all plus signs.

\section{Constructing equations of the refined plate theory}

A complete system of equations in the plate theory consists of the equilibrium equations, geometrical relations, and constitutive relations. The equilibrium equations and geometrical relations between strains and displacements are the same in all plate theories because they are mathematically strict. The difference lies in the elasticity relations. Therefore, we will only consytuct and discuss two-dimensional elasticity relations.

For the elasticity equations (1) - (4), we will do a scale extension typical of the asymptotic methods, with respect to the coordinates

For the elasticity equations (1) - (4), we will do a scale extension typical of the asymptotic methods, with respect to the coordinates

$$
\frac{\partial}{\partial x_{i}}=\eta^{-s} \frac{1}{R} \frac{\partial}{\partial \xi_{i}}, \quad \frac{\partial}{\partial x_{3}}=\eta^{-1} \frac{1}{R} \frac{\partial}{\partial \varsigma} ;
$$

We except for the plate the following asymptotics

$$
\frac{u_{\mathrm{i}}}{R}=\eta^{s-1} u_{i^{*}}, \frac{u_{3}}{R}=\eta^{2 s-2} u_{3^{*}}, \frac{\sigma_{i i}}{E}=\eta^{-1} \sigma_{i i^{*}}, \frac{\sigma_{i j}}{E}=\eta^{-1} \sigma_{i j^{*}}, \frac{\sigma_{i 3}}{E}=\eta^{-s} \sigma_{i 3^{*}}, \frac{\sigma_{33}}{E}=\eta^{1-2 s} \sigma_{33^{*}}
$$

Here all quantities with asterisks in (7) are of the same order and are dimensionless.

Substituting formulas (6), (7) in the equations (1) - (4), we obtain the following equations:

$$
\begin{gathered}
\frac{\partial \sigma_{i i^{*}}}{\partial \xi_{i}}+\frac{\partial \sigma_{i j^{*}}}{\partial \xi_{j}}+\frac{\partial \sigma_{i 3^{*}}}{\partial \varsigma}=0, \frac{\partial \sigma_{13^{*}}}{\partial \xi_{1}}+\frac{\partial \sigma_{23^{*}}}{\partial \xi_{2}}+\frac{\partial \sigma_{3}}{\partial x_{3}}=0 . \\
e_{i i^{*}}=\frac{\partial u_{i^{*}}}{\partial \xi_{i}}, \quad e_{i j^{*}}=\frac{\partial u_{i^{*}}}{\partial \xi_{j}}+\frac{\partial u_{j^{*}}}{\partial \xi_{i}}, \quad g_{i^{*}}=\frac{\partial u_{3^{*}}}{\partial \xi_{i}} . \\
\sigma_{i i^{*}}=\frac{1}{\left(1-v^{2}\right)}\left(e_{i i^{*}}+v e_{j j^{*}}\right)+\eta^{2-2 s} \frac{v}{(1-v)} \sigma_{33^{*}}, \quad \sigma_{i j^{*}}=\frac{1}{2(1+v)} e_{i j^{*}} . \\
\frac{\partial u_{i^{*}}}{\partial \varsigma}=-g_{i^{*}}+\eta^{2-2 s} 2(1+v) \sigma_{i 3^{*}}, \quad \frac{\partial u_{3^{*}}}{\partial \varsigma}=\eta^{4-4 s} \sigma_{33^{*}}-\eta^{2-2 s} v\left(\sigma_{11^{*}}+\sigma_{22^{*}}\right) .
\end{gathered}
$$

In order to reduce approximately the $3 \mathrm{D}$ elasticity equations to two-dimensional ones, we integrate equations (8) - (11) to $\varsigma$. Then all the needed quantities (displacements, stresses, and strains) will be represented as polynomials in $\varsigma$. The remaider of the series presented are those greatest terms are small quantities of order $\varepsilon_{r}$, where 


$$
\varepsilon_{r}=(h / R)^{4-4 s}
$$

We will neglect the terms of order $\varepsilon_{r}$ in the resultant asymptotic expansions and write the latter as

$$
\begin{gathered}
u_{i^{*}}=u_{i, 0}+\varsigma u_{i, 1}+\eta^{2-2 s} \varsigma^{2} u_{i, 2}+\eta^{2-2 s} \varsigma^{3} u_{i, 3} \quad\left(e_{i l^{*}}, e_{i j^{*}}, \sigma_{i l^{*}}, \sigma_{i j^{*}}\right) ; \\
u_{3^{*}}=u_{3,0}+\eta^{2-2 s} \varsigma u_{3,1}+\eta^{2-2 s} \varsigma^{2} u_{3,2} \quad\left(g_{i^{*}}\right) ; \\
\sigma_{i 3^{*}}=\sigma_{i 3,0}+\varsigma \sigma_{i 3,1}+\varsigma^{2} \sigma_{i 3,2}+\eta^{2-2 s} \varsigma^{3} \sigma_{i 3,3}+\eta^{2-2 s} \varsigma^{4} \sigma_{i 3,4} ; \\
\sigma_{33^{*}}=\sigma_{33,0}+\varsigma \sigma_{33,1}+\varsigma^{2} \sigma_{33,2}+\varsigma^{3} \sigma_{33,3}+\eta^{2-2 s} \varsigma^{4} \sigma_{33,4}++\eta^{2-2 s} \varsigma^{5} \sigma_{33,5} ;
\end{gathered}
$$

In the parentheses on the right of the formulas, we enumerate the quantities whose asymptotic expansions have the same form.

We substitute formulas (13) - (16) into equations (8) - (11). By equating the coefficients at the same powers of $\varsigma$, we get a system of 65 differential equations with 65 unknowns. The system can be integrated without resorting to the traditional notations of the plate theory, i.e., forces, moments, displacements, and strains of the middle plane. Nevertheless, the transition is necessary if we are to make a comparison with the other theories.

We apply the notation of plate theory to our equations. The displacements $v_{i}$ and $w$ of the middle plane are related to the three-dimensional displacement $u_{i}$ and $v_{3}$ as

$$
v_{i}=\left.u_{i}\right|_{x_{3}=0}, w=\left.u_{3}\right|_{x_{3}=0}
$$

In addition to the displacement of the middle plane in the refined theory should be taken into account the transverse shear deformation $\psi_{i 3}$.

We express the forces and the moments through the stresses

$$
T_{i}=\int_{-h_{0}}^{+h} \sigma_{i i} d x_{3}, \quad \mathrm{~S}_{i j}=\int_{-h_{0}}^{+h} \sigma_{i j} d x_{3}, \quad G_{i}=\int_{-h_{0}}^{+h} \sigma_{i i} x_{3} d x_{3}, \quad \mathrm{H}_{i j}=\int_{-h_{0}}^{+h} \sigma_{i j} x_{3} d x_{3}, N_{i}=\int_{-h_{0}}^{+h} \sigma_{i 3} d x_{3}
$$

Leaving out the simple, but cumbersome, transformations, we obtain the elasticity relations and geometrical relations between strains and displacements of the middle plane for refined plate theory

$$
\begin{gathered}
T_{i}=\frac{2 E h}{1-v^{2}}\left(\varepsilon_{i}+v \varepsilon_{j}\right)+ \\
+\left\{D \frac{v}{2(1-v)}\left(\frac{\partial^{2}}{\partial x_{i}^{2}}+v \frac{\partial^{2}}{\partial x_{j}^{2}}\right)\left(\varepsilon_{1}+\varepsilon_{2}\right)+\frac{2 v h}{1-v} p+\frac{2 h^{2}}{3(1-v)}\left(\frac{\partial n_{i}}{\partial x_{i}}+v \frac{\partial n_{j}}{\partial x_{j}}\right)+\frac{2 v h^{2}}{3(1-v)}\left(\frac{\partial n_{1}}{\partial x_{1}}+\frac{\partial n_{2}}{\partial x_{2}}\right)\right\} \\
\mathrm{S}_{12}=\mathrm{S}_{21}=S=\frac{E h}{1+v} \omega+\left\{\frac{v D}{2} \frac{\partial^{2}}{\partial \mathrm{x}_{1} \partial x_{2}}\left(\varepsilon_{1}+\varepsilon_{2}\right)+\frac{h^{2}}{3}\left(\frac{\partial n_{1}}{\partial x_{2}}+\frac{\partial n_{2}}{\partial x_{1}}\right)\right\}
\end{gathered}
$$




$$
\begin{aligned}
& G_{i}=-D\left(\kappa_{i}+v \kappa_{j}\right)+ \\
& +\frac{h^{2}}{5(1-v)}\left\{-D v\left(\frac{\partial^{2}}{\partial x_{i}^{2}}+v \frac{\partial^{2}}{\partial x_{j}^{2}}\right)\left(\kappa_{1}+\kappa_{2}\right)+4\left(\frac{\partial \mathrm{N}_{i}}{\partial x_{i}}+v \frac{\partial \mathrm{N}_{\mathrm{j}}}{\partial x_{j}}\right)-2 v\left(\frac{\partial \mathrm{N}_{1 i}}{\partial x_{1 i}}+\frac{\partial \mathrm{N}_{2}}{\partial x_{2}}\right)-\frac{4 h}{3}\left(\frac{\partial m_{i}}{\partial x_{i}}+v \frac{\partial m_{\mathrm{j}}}{\partial x_{j}}\right)+\frac{2 v h}{3}\left(\frac{\partial m_{1}}{\partial x_{1}}+\frac{\partial m_{2}}{\partial x_{2}}\right)\right\} \\
& \mathrm{H}_{12}=\mathrm{H}_{21}=\mathrm{H}=-D(1-v) \tau+ \\
& +\left\{-D \frac{v h^{2}}{10} \frac{\partial^{2}}{\partial \mathrm{x}_{1} \partial x_{2}}\left(\kappa_{1}+\kappa_{2}\right)+\frac{2 h^{2}}{5}\left(\frac{\partial N_{1}}{\partial \mathrm{x}_{2}}+\frac{\partial N_{2}}{\partial x_{1}}\right)-\frac{2 h^{3}}{15}\left(\frac{\partial m_{1}}{\partial x_{2}}+\frac{\partial m_{2}}{\partial x_{1}}\right)\right\} \\
& N_{i}=\frac{2 \mathrm{Eh}}{3(1+v)} \psi_{i 3}+\frac{2 h}{3} m_{i} \\
& \varepsilon_{i}=\frac{\partial v_{i}}{\partial x_{i}}, \quad \omega=\frac{\partial v_{2}}{\partial x_{1}}+\frac{\partial v_{1}}{\partial x_{2}}, \quad \kappa_{i}=\frac{\partial^{2} w}{\partial x_{i}^{2}}, \quad \tau=\frac{\partial^{2} w}{\partial \mathrm{x}_{1} \partial x_{2}}
\end{aligned}
$$

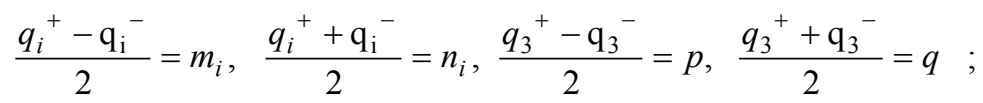

The obtained system of equations of refined plate theory has the sixteen order. The plane problem and the bending problem with the elasticity relations (18), (19) and (20) (22) respectively have an eighth order.

\section{On the error of refined Timoshenko's plate theory}

We write out the refined relations of elasticity of Tymoshenko [3] for bending problem taking into account the transverse shear deformation

$$
\begin{gathered}
G_{i}=-\mathrm{D}\left(\frac{\partial^{2} w}{\partial x_{i}{ }^{2}}+v \frac{\partial^{2} w}{\partial x_{j}{ }^{2}}\right)+\frac{4 h^{2}}{5} \frac{\partial N_{i}}{\partial x_{i}}-\frac{2 h^{2}}{5} \frac{v}{1-v} q \\
\mathrm{H}_{i j}=-(1-v) D \frac{\partial^{2} w}{\partial x_{i} \partial x_{j}}+\frac{2 h^{2}}{5}\left(\frac{\partial N_{i}}{\partial x_{i}}+\frac{\partial N_{j}}{\partial x_{j}}\right) \\
N_{i}=\frac{5}{12} \frac{2 E h}{1+v} \psi_{i 3}
\end{gathered}
$$

The system of differential equations for the bending problem has sixth order.

Comparing the relations (20) - (22) and (25) - (27), we see that the elasticity relations of Timoshenko lack a number of small terms of the same order of magnitude as the transverse shear deformation. It means that the error of the Tymoshenko theory is exactly the same as that of the Kirchhoff theory. The same asymptotically inconsistent are the theories of plates and shells of E. Reissner and Naghdi [4 -6], taking into account the transverse shift. It was shown by the asymptotic method in [7]. Despite the fact that it is proved that the theory of plates and shells, only the transverse shear deformation, does not 
specify the Kirchhoff theory, the number of works based on these theories does not decrease. (see, for example, [8]).

\section{References}

1 V.V. Novozhilov, R.M. Finkelshtein, PMM, 38, 5-6, (1943)

2 A.L. Golden'veizer, The Theory of Thin Elastic Shells (Nauka, Moscow, 1976)

3 S.P. Timoshenko, S. Woinowsky-Krieger, Theory of Plates and Shells (McGraw-Hill New York, 1959)

4 E. Reissner, Quart.Appl. Math. 5, 1 (1947)

5 E. Reissner,. J. Math. and Phys. 23, 4, 184-191 (1944)

6 N.N. P.M. Naghdi, Quart.of J. Appl. Math. 14, 4, (1957)

7 N.N. Rogacheva, J. Appl.. and Mech. 38, 6, 1063-1071 (1974)

8 I.A. Maneviich, Z. Kolakovsky, Prikl. Mech. 50, 2, 105-114 (2014) 